\title{
Multi-rotors: A Revolution In Unmanned Aerial Vehicle
}

\author{
Kanaiya Agrawal ${ }^{1}$, Punit Shrivastav ${ }^{2}$ \\ ${ }^{1,2}$ P.I.E.T (Vadodara) affiliated to GTU (Ahmedabad), Vadodara, India
}

\begin{abstract}
Multi- rotor technology is the advanced technology for wireless drones. Here in this paper it is discussed about different types of copter with different rotors have vividly advantages and applications. They were available in single, double, triple, quad, hex, octa, copters. Multi-copters use brushless motors rather than any other simple dc motors. Multi-copters are most probably used nowadays for variety of its applications in communication purposes as wells as in video surveillance and in defense also. Here, comparison is also made between different types of copters and a suitable conclusion is made out of their applications
\end{abstract}

Keywords: rotors, brushless motors, multi-copters, yaw

\section{Introduction}

Multi-copters are most probably used wireless drones used for various purposes. The multi-copters are one of the most complex flying machines due to its versatility to perform many types of tasks. The first multi-rotor helicopter with fixed pitch blades rotors was proposed in 1923 by De Bothezat, but technology at that time was not ready for applicable construction of such type of machine. The main problem was an instability of the vehicle and thus pilot workload which was too high.[1] Since then the development of light batteries, brushless actuators and MEMS sensors allowed considerable improvement in construction and control of this type of helicopter. Today's multi-copters are not only radio-controlled toys but they are robust and simple helicopters that are able to bear nearly two kilograms payload [2]. They have started to be used as UAV (Unmanned Aerial Vehicles) or aerial robots mainly for identification purposes. Also, Military applications are ready to come soon. Multirotors are to be considered as most stable wireless drones. Multi-rotor helicopters (tri, quad, hexa, octa, etc) become popular research platforms for UA Vs (Unmanned Aerial Vehicles). Among them, quadrotor UAVs have dynamically simple and strong structure [14], so they are used widely for various experiments. Since a hexa-copter or an octa-copter can carry heavier payload than a quadrotor, they are used for aerial photograph or even broadcasting.[15].. They are available in various different types such as bi-copters, tricopters, quad-copters, penta-copters, hexa-copters, octacopters, out of all these copters hexa and octa copters are considered to be the most stable drones.

\section{Types of Multi-Rotors}

\subsection{BI-COPTERS}

The BiCopter has two motors which can be moved by servos. It is enriched with two servo motors and hence considered as the cheapest multicopter, but it is the least stable and is difficult to tune. It's also the least robust and has less lifting power because it has only two rotors. [3]. If one of the rotors fails to rotate then copter will not be able to move further which was its biggest limitation.

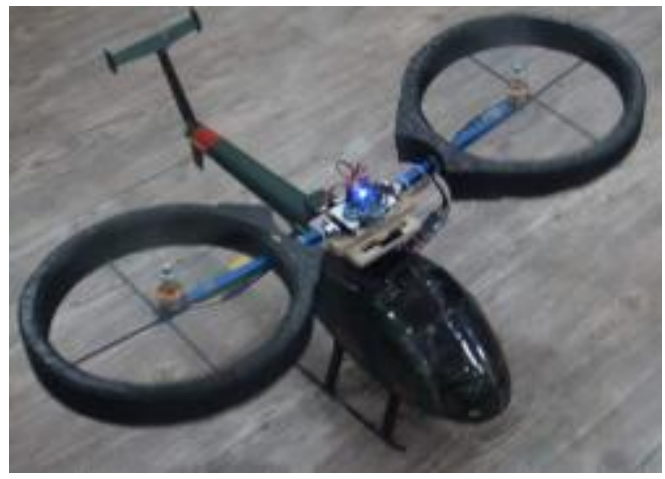

Figure 1: Bi-copter[4]

\subsection{TRI-COPTERS}

Tricopters are considered to be the least expensive type of multirotor because they have the fewest motors i.e only three motors. They also require a servo motors which are much less expensive than brushless motors. Another advantage of the tricopter is that it has the widest angle (120 degrees) between the front two motors, making it easy to do videography in air while the propellers remains out of shot.[5]

The Tricopter has three motors which are arranged in a " $Y$ " shape usually 120 degrees apart, or sometimes they are in " $T$ " shape too. Two propellers which are on the front arms pointed to the sides or slightly forwards, and one arm backwards.[3]. The rear motor can be tilted laterally using a support. This supportive mechanism provides yaw control for the craft.[5].

Tri-copter is stable compared to bi-copter, but it is highly unstable compared to other types of multi-copters, also if one of its rotors fails to rotate than copter will not be able to move further. 


\section{International Journal of Science and Research (IJSR) \\ ISSN (Online): 2319-7064}

Index Copernicus Value (2013): 6.14 | Impact Factor (2014): 5.611

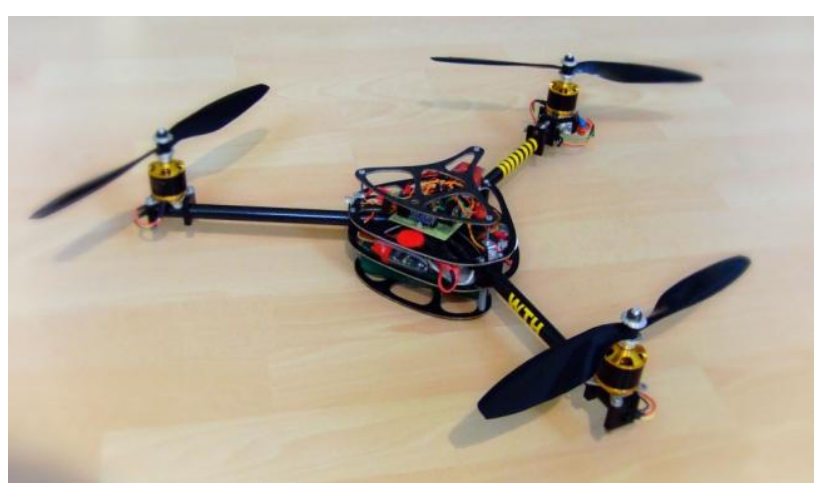

Figure 2: Tri-copter[6]

As Tricopter consists of only three motors, it has low lifting power. Also because of its yaw mechanism it is less stable.

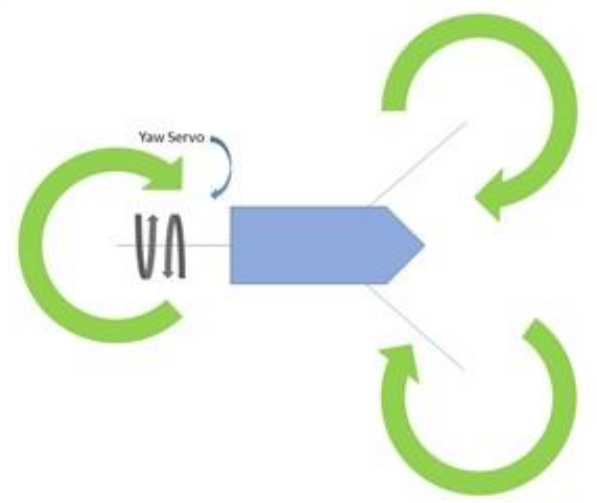

Figure 3: Servo on rear motor for yaw control

Advantages of tri-copter

- less Motor

- Decreased Weight

- Longer Battery Life

- Cheaper

\subsection{QUAD-COPTERS}

A quadcopter, also called a quadrotor helicopter, [7] is a multirotor helicopter that is lifted and propelled by four rotors. A quadcopter consists of 4 motors which are mounted on four symmetrical frames, each arm is 90 degree apart specially (for X4 configuration). There are two sets of Clockwise $(\mathrm{CW})$ and Counter Clockwise $(\mathrm{CCW})$ propellers mounted on the motors to create opposite force to balance it. The configuration of quadcopter is the most popular type stable, simplest and easiest to understand [3].

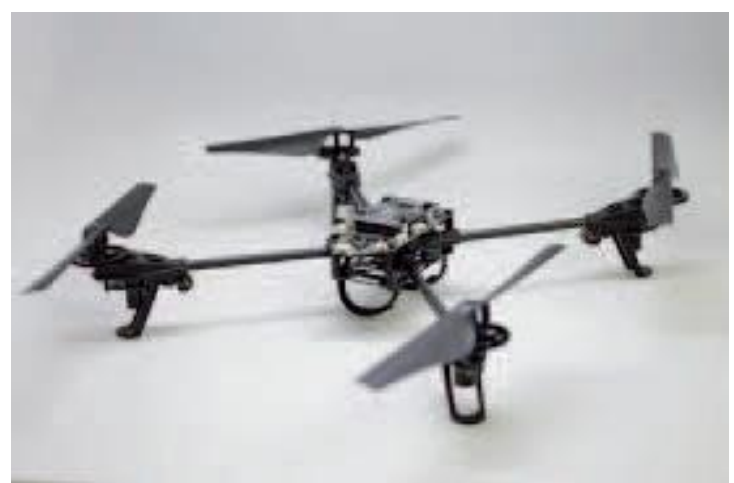

Figure 4: Quad-copter[8]
Quad-copter's movement depends on the speed of its motor. It is compatible that all the 4 motors should rotate with same speed so that it can create same lifting power hover with balance. Also, the distribution of mass should be prevalent in quad-copter and its mass point should be at the center.[9].

The main advantages of quadcopters over other copters are that the quadcopters do not require mechanical linkages to vary the rotor blade pitch angle as they stroll or spin. This makes design of quadcopter simple.[10] Also, the use of 4 rotors allows each individual rotor to have a smaller diameter than the equivalent helicopter rotor, due to which they possess less kinetic energy during flight. This reduces the damage of hitting rotors anything. For small-scale unmanned aerial vehicle (UAV), this makes the vehicles safer. Some of the quadcopters have frames that enclose the rotors, permits the flights to travel from more testing environments, with less risk of damaging the copter.[11].

\section{$\mathrm{X}$ Configuration}

The $\mathbf{X}$ configuration of quadcopter is the most popular quadcopter design, which makes it the most popular multirotor design overall. As in the $\mathrm{X}$ configuration the camera can point out between the front two motors makes it helpful to be used for aerial photography/videography. [5]

\section{+ Configuration}

The + configuration is easier to control compared to $\mathrm{X}$ configuration and can fly like an airplane. Therefore it is used in acrobatic flying.[5]

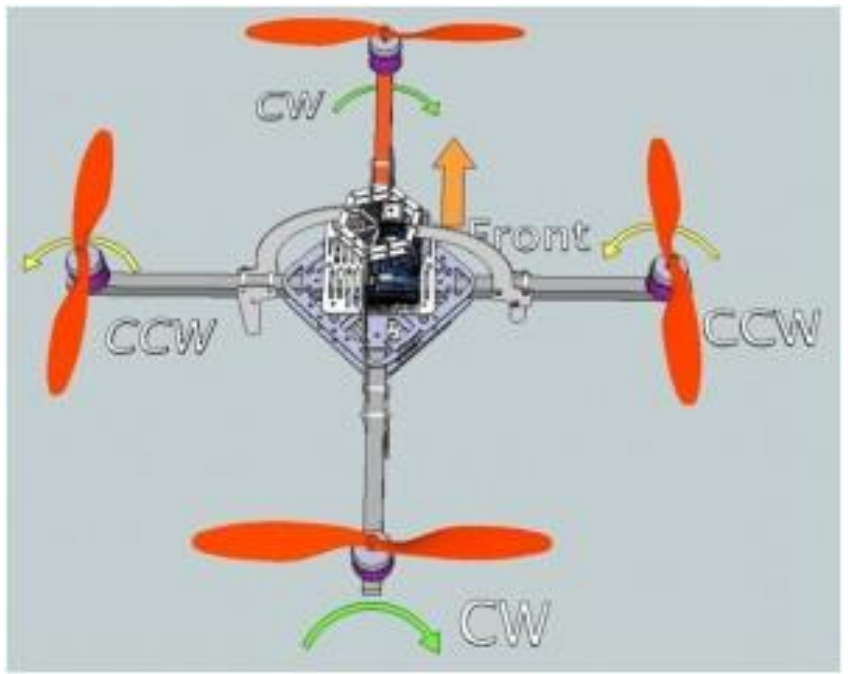

Figure 5: Rotation for quad copter in + configuration [3]

\section{Y4 Configuration}

Another useful configuration is Y4 quadcopter which seems just as tri-copter, but instead of using servo motor on the rear motor, Y4 copters have a another brushless motor mounted underneath the first. Thus, by altering the relative speed of the two rear motors yaw control is achieved. While Y4 copters fly the same as tricopters, but they have more lifting power and are more robust than tri-copter [5]. 


\section{International Journal of Science and Research (IJSR) \\ ISSN (Online): 2319-7064}

Index Copernicus Value (2013): 6.14 $\mid$ Impact Factor (2014): 5.611

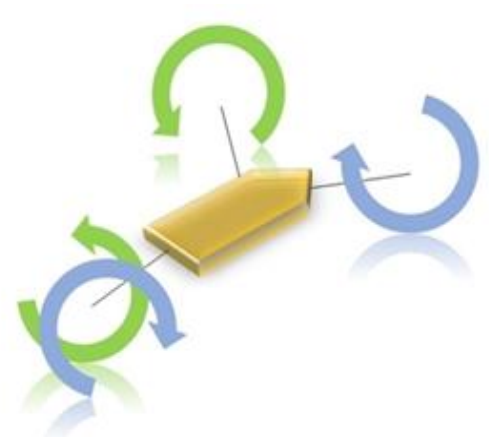

Figure 6: Y4 configuration for quad copters

Advantages of quadcopter:

- Brushless motors are used

- Long battery life

- Nice stability

\subsection{PENTA-COPTERS}

Pentacopters have five motors. There is not much information available about penta-copter because it is not as popular as other types of copters due to possible underlying issues.[3]. Pentacopters are very uncommon though, and are considered to be the worst multirotor design.[5].

One main advantage of the pentacopter is the wide angle of the two front arm which allows the propellers to stay out of the camera as far as possible.[3].

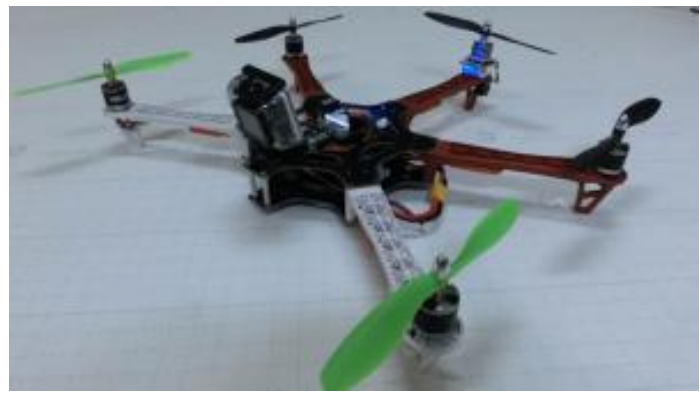

Figure 7: Penta copter [3]

One another advantage of pentacopters is the wide angle of the front arms, which makes it easy to shoot aerial photos and videos without the props making it into frame. Also, pentacopters have more lifting power than tricopters or quadcopters.[5].

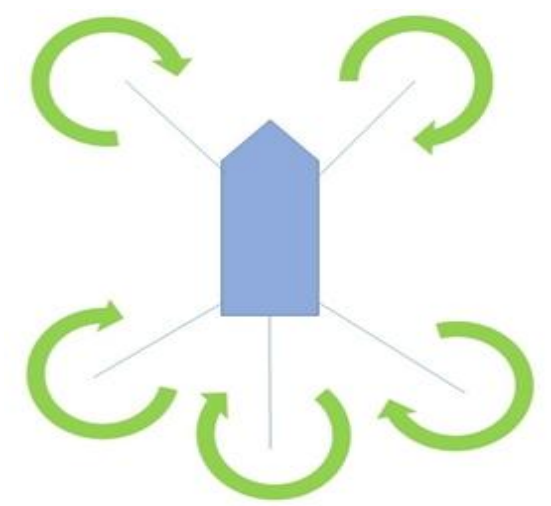

Figure 8: Rotation configuration for penta-copters

\subsection{HEXA-COPTERS}

The term Hexa-copter is a VTOL (Vertical Take-Off and Landing) aerial vehicle belonging to the class of multirotor helicopters. They differ from the standard helicopters in using rotors with fixed pitch, thus as the blades rotate their rotor pitch does not vary. Hexacopter consists of six rotors [12].

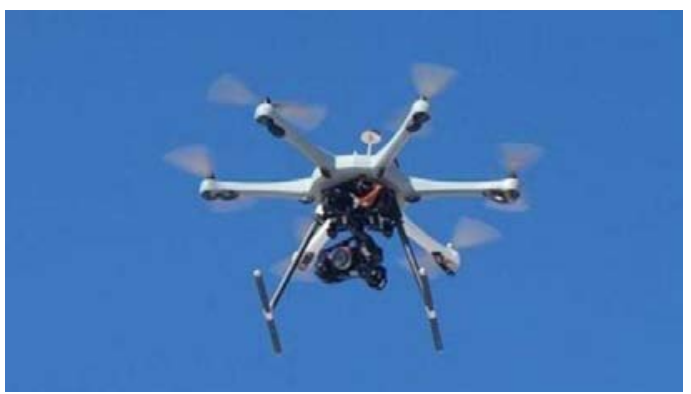

Figure 8: Hexacopter with six rotors [13]

The hexacopter has six motors which are mounted on 6 symmetrical frames making 120 degree of angle from eachother. They have three sets of CW (clockwise) and $\mathrm{CCW}$ (counter clockwise) propellers. It's very similar to the quadcopter, but due to larger number of motors it provides more lifting power. The main advantage behind hexa-copter is that if one of its motor fails then also it can still fly in air.[3].

Hexacopters has more lifting power than quadcopter inspite of having similar flight mechanics. Due to extra motor they they have more lifting power. This makes it easy to carry camera equipment more implex than an average action camera, like high-end DSLRs (digital single-lens reflex).[5].

There are mainly 3 different types of configuration of hexacopter:

\section{a. X Configuration/+ Configuration}

Like quadcopters, hexacopters can be configured either in $\mathbf{X}$ or + mode. In both of these designs, the rotors are arranged with equal spacing around the body. The only difference between X mode and + mode is that, in X mode two of the motors face front while in + mode one of the motor faces front. Like quadcopters, + mode is most commonly used for acrobatic flying and $\mathbf{X}$ mode is most commonly used for aerial videography or photographic purpose. [5].

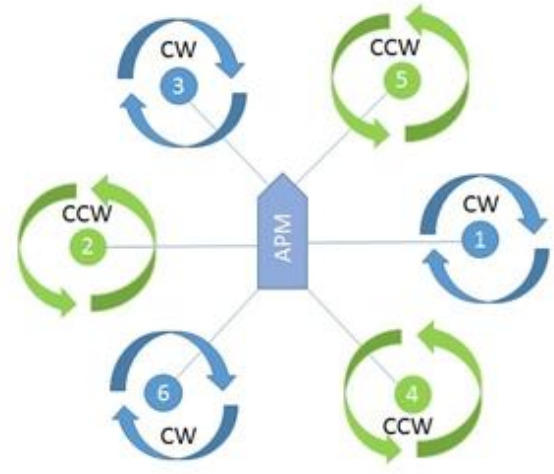

Figure 9: $\mathrm{X}$ configuration of hexacopt 


\section{International Journal of Science and Research (IJSR) \\ ISSN (Online): 2319-7064 \\ Index Copernicus Value (2013): 6.14 | Impact Factor (2014): 5.611}

a. Y6

Y6 copters are as similar as tricopters, the only difference is thathexacopter contains two motors on each arm which is mounted on top of other. The pairs of motors stroll in opposite directions and just balance the forces and prevent an unintended yaw. Y6 copters are more compact than other hexacopters but are slightly less efficient.[5].

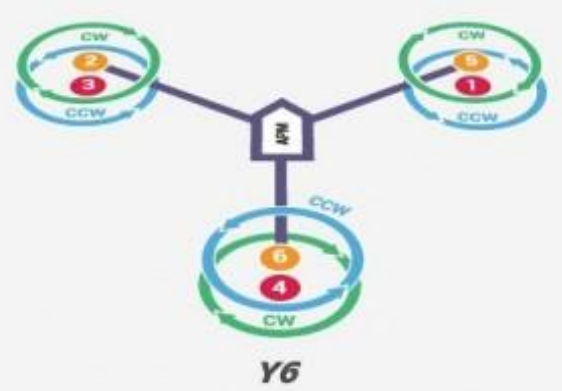

Figure 10: Y6 configuration of hexa copter [3]

Advantages

- Higly stable

- Can also stay in air if one of its rotors fails.

Disadvantages

- Heavy

- Larger

- More expensive

\subsection{OCTA-COPTERS}

An octa-copter consists of 8 rotors which consists of four sets of $\mathrm{CW}$ (clockwise) and remaining four sets of $\mathrm{CCW}$ (anticlockwise) propellers. It's an upgrade version of the hexacopter with even more lifting capacity. If one or two motors of octa-copter fail, it should still be supported by other motors and able to land safely. Due to this property of octa-copter it is considered to be the most reliable among the other multi-rotors.[3].

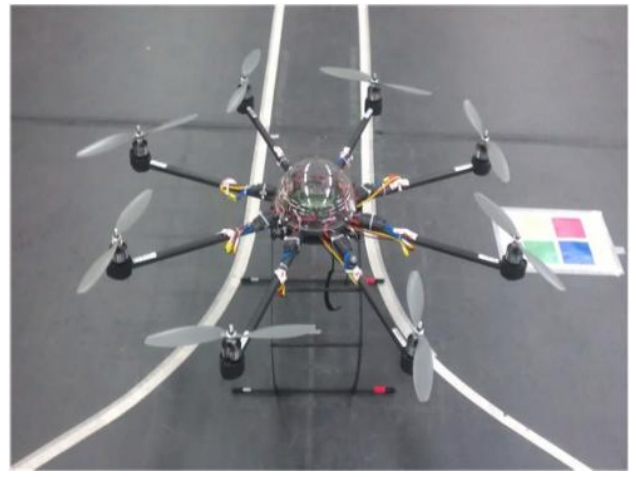

Figure 11: Octacopter with eight rotors [15]

Octocopters are very similar to hexacopters. They have more lifting power than hexa-copters and quad-copters. Just like hexa-copters octa-copter can also be build in three different configurations.[5]

\section{Configuration/+ Configuration}

Likewise in hexacopters, octocopters can also be configured as $\mathbf{X}$ or + mode. In both of these designs, the rotors are arranged with equal spacing around the body. The only difference between $\mathrm{X}$ mode and + mode is that, in X mode two of the motors face front while in + mode one of the motor faces front. Like hexa-copters, + mode is most commonly used for acrobatic flying and $\mathbf{X}$ mode is most commonly used for aerial photography/videography.[5].

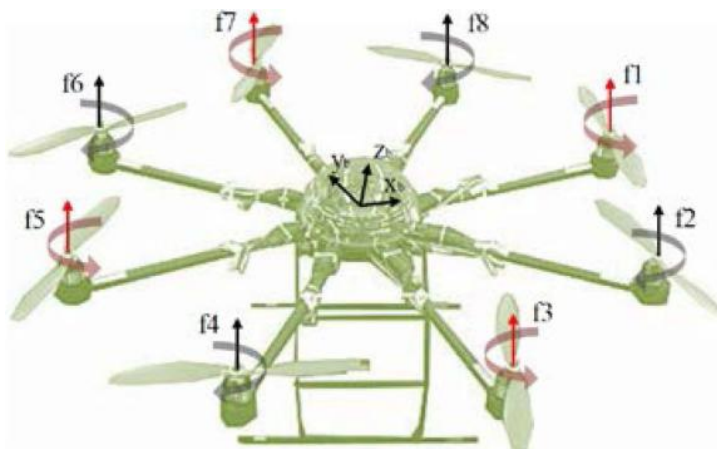

Figure 12: Hexa-copter with $X$-configuration [15]

b. $\mathrm{X8}$

X8 copters are similar to quadcopters, but X8 copters have two motors on each arm which is mounted on top of the other. In such a way, X8 copters are something similar to Y6 copters. X8 copters have highest lifting power than hexacopters and quadcopters because they have number motors than any other copters. [5].

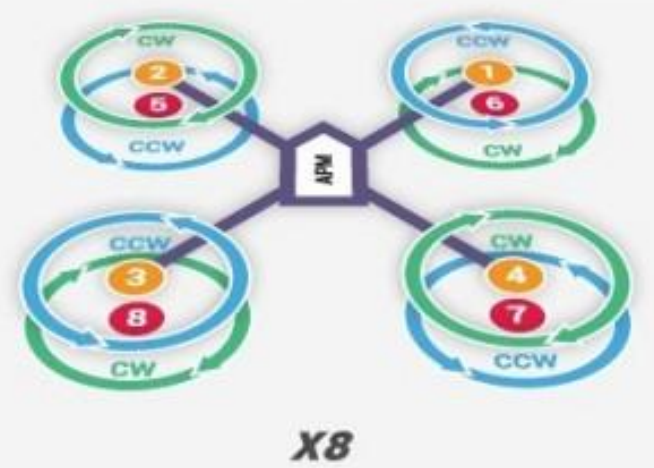

Figure 13: Octa-copter with $\mathrm{X} 8$ configuration [3]

\section{Comparison}

Table 1

\begin{tabular}{|c|c|c|c|c|c|}
\hline S No. & $\begin{array}{c}\text { Types of } \\
\text { copters }\end{array}$ & $\begin{array}{c}\text { No. of } \\
\text { rotors }\end{array}$ & Stability & $\begin{array}{c}\text { Lifting } \\
\text { power }\end{array}$ & Cost \\
\hline 1. & Bi-copter & 2 & Less stable & Very low & $\$ 150$ \\
\hline 2. & Tri-copter & 3 & Less stable & Very low & $\$ 180$ \\
\hline 3. & Quad-copter & 4 & stable & average & $\$ 350$ \\
\hline 4. & Penta-copter & 5 & Less stable & high & $\$ 430$ \\
\hline 5. & Hexa-copter & 6 & Higly stable & high & $\$ 700$ \\
\hline 6. & Octa-copter & 8 & Most stable & Very high & $\$ 1200$ \\
\hline
\end{tabular}

\section{Application of Multi-Rotors}

Search and Rescue: Would require night vision and infrared optics 


\section{International Journal of Science and Research (IJSR) \\ ISSN (Online): 2319-7064}

Index Copernicus Value (2013): 6.14 | Impact Factor (2014): 5.611

HazMat: Instead of sending people to read the placard, send a quad-copter

Police: Similar to search and rescue, but for the "bad guys" Code Enforcement/Inspections: Building and bridge inspection without placing a person on a ladder or other potentially dangerous situation.

Emergency: Situation awareness and Damage assessment after disaster.

Fire : Better view of incident, Infrared can locate area of fire.

\section{Future Scope}

With the increasing advantages of multi-rotors more improvement can be done in this field. Multi-copters with more battery life can be developed which will remain in air for longer time. Weight of the copters will be reduced to make it more efficient and reliable. Voice command can also be added to make it more sophisticated. They can work in the remote areas where man could not be able to reach and thus makes much hazardous work to be easy.

\section{Conclusion}

Here we have discussed various types of multi-rotors with their advantageous and applications. We have studied about their different configuration which makes them stable in air. We have also estimated their cost and stability, due to which we are able to predict out the better multi-rotor as per their application. They are the most stable currently used unmanned aerial vehicles. They are the unmanned robot which has to be proved more helpful surveillance and many hazardous applications. Out of all multi-rotors Hexa-copter and Octa-copter are considered to be the most stable one, also Hexa-copter is preferred most probably due to its less price compared to Octa-copter.

\section{References}

[1] Radek Baránek, František Šolc "Modelling and Control of a Hexa-copter" IEEE $201213^{\text {th }}$ international Carpathian control conference (ICCC) High Tatras Slovakia.2012/05/28.

[2] POUNDS, P., MAHONY, R., CORKE, P. Modelling and Control of a Quad-Rotor Robot. In Proceedings of the Australasian Conference on Robotics and Automation, Auckland, New Zealand: Australian Robotics \& Automation Association, Dec. 2006, p. 501510.

[3] http://blog.oscarliang.net/types-of-multicopter/

[4] http://www.multiwii.be/sites/default/files/bimultirotor.pn $\mathrm{g}$

[5] http://blacktieaerial.com/2014/05/13/types-of-multirotor/

[6] http://www.villalachouette.de/william/krims/tricopter/we bsitepics/tricopter_dlx_detail0.jpg

[7] Hoffmann, G.M.; Rajnarayan, D.G.; Waslander, S.L.; Dostal, D.; Jang, J.S.; Tomlin, C.J. (November 2004). "The Stanford Testbed of Autonomous Rotorcraft for Multi Agent Control (STARMAC)".In the Proceedings of the 23rd Digital Avionics System Conference. Salt Lake City, UT. pp. 12.E.4/1-10.
[8] Professor Dr. Götz Winterfeldt, Christina Hahne“ Controlling Quad-Copters"2014 IEEE Global Engineering Education Conference (EDUCON) 3rd-5th April 2014, Military Museum and Cultural Center, Harbiye, Istanbul, Turkey.

[9] Stevie Jeremia, Endrowednes Kuantama, Julinda Pangaribuan "Design and Construction of RemoteControlled Quad-copter Based on STC12C5624AD" 2012 International Conference on System Engineering and Technology IEEE September 11-12, 2012, Bandung, Indonesia.

[10] Pounds, P.; Mahony, R.; Corke, P. (December - 2006). "Modeling and Control of a Quad-Rotor Robot". In the Proceedings of the Australasian Conference on Robotics and Automation. Auckland, New Zealand.

[11] Hoffman, G.; Huang, H.; Waslander, S.L.; Tomlin, C.J. (20-23 August 2007). "Quadrotor Helicopter Flight Dynamics and Control: Theory and Experiment". In the Conference of the American Institute of Aeronautics and Astronautics. Hilton Head, South Carolina.

[12] Radek Baránek, František Šolc "Modelling and Control of a Hexa-copter" IEEE $201213^{\text {th }}$ international Carpathian control conference (ICCC) High Tatras Slovakia.2012/05/28.

[13] http://www.uasvision.com/wpcontent/uploads/2013/10/Kang-Ben-Hexacopter.jpg

[14]Hyon Lim, Jaemann Park, Daewon Lee, and H.Jin Kim, "Build your own quadrotor: Open-source projects on unmanned aerial vehicles, " IEEE Robotics \&Automation Magazine, Vol. 7, No.3, pp. 33-45, 2012.

[15] Hyeonbeom Lee, Hyon Lim, Suseong Kim Daewon Lee, H. Jin Kim "Control of an Octa-copter from Modeling to Experiments" IEEE2014 international symposium on robotics (ISR) Seoul Korea (south).

\section{Author Profile}

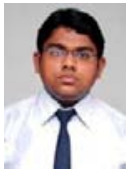

Kanaiya Agrawal received the B.E degree in Electronics Engineering from Bhagwaan Mahaveer College of Engineering And Technology affiliated to GTU in 2013, currently pursuing his Masters Degree in communication from Parul Institute of engineering and technology (2014-2016).His researches mostly based on wireless communication and networking security

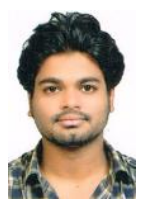

Punit Shrivastav received the B.E degree in Electronics and Telecommunications from D.Y Patil College of engineering and technology (Pune) in 2014 currently pursuing his Masters Degree in communication from Parul Institute of engineering and technology (2014-2016). 